\title{
Construction of models for bounded price processes: the case of the HKD exchange rate
}

\author{
Yee Hong Ben \& Nikolai Dokuchaev \\ Department of Mathematics \& Statistics, Curtin University, \\ GPO Box U1987, Perth, 6845 Western Australia
}

June 5, 2015

\begin{abstract}
This paper discusses construction of evolution models for financial time series evolving within a given interval. We calibrated a model for the case of the USD/HKD exchange rate after the separation of strong and weak side convertibility undertakings, in which the rate is confined to a specified corridor. This process represents an interesting example of a tradable bounded process. A 1D model was able to replicate the bounded distribution of the process, but a 2D model better captured dynamics as measured by the volatility without losing features of the 1D model. We briefly consider the ergodic properties of these models.
\end{abstract}

JEL classification: C14, C51, C53, C58, G10

Keywords: currency corridor; bounded financial time series; 2D Markov model

\section{Introduction}

This paper suggests an evolution model for financial time series in the presence of reflecting boundaries or other factors that enforce some restrictions on its movements. These processes are not very common in financial modelling; however, there are some important examples of these processes such as volatility indices, and foreign exchange under a currency corridor. The model suggested in this paper was tested and calibrated for the USD/HKD exchange rate after the separation of strong and weak side convertibility undertakings. In 1983, prior to May 2005, the HKD was taken off a free float and pegged at a fixed rate to the USD under the linked exchange rate system by a single convertibility undertaking at 7.80. From May 2005, this was separated into the strong and weak side convertibility undertakings to form a target zone of $7.75-7.85$. Effectively, the result is that it functions as a currency band in which exchange rates are maintained within a target zone; that is, the rate is expected to remain between an upper and lower bound [1]. We consider only this latter period.

This particular exchange rate is noteworthy as the HKD is a tradable asset which has successfully maintained its exchange rate with the USD under a currency band for a significant period of time. As far as we know, this represents a unique example of a tradable asset with price

Preprint of an author accepted manuscript following peer review accepted for publication in the Annals of Financial Economics (C) 2015 World Scientific Publishing Company

(Journal URL: http://www.worldscientific.com/worldscinet/afe) 
fluctuations within a predetermined range. Of the currencies pegged in such a manner, none can match the liquidity or tradability of the HKD with the status of Hong Kong as a financial hub. The HKD is thus a rare and unique tradable asset for which trading strategies operating under such a bounded dynamic may be considered [2]. According to modern portfolio theory, existence of a tradable asset with mean reverting price dynamics will lead to unusual speculative opportunities or even arbitrage opportunities in spite of the unpredictability of prices $[3,4,5]$. The property of boundedness is stronger than the mean reversion property in the sense that instead of simply being assured that a process will eventually return to a mean, it is assured that a bounded process will never leave a particular interval. As such, the same arguments leading to speculative or arbitrage opportunities still apply.

It can be noted that although there are traded commodities whose price variations are similarly confined to a band, such price enforcement is usually accompanied by trading restraints which limit liquidity; such situations therefore do not represent an interesting case from the point of view of arbitrage theory. The USD/HKD exchange on the other hand, is a consequence of the money market, which having some of the highest trading volumes and liquidities of all financial markets, could present an interesting case.

We consider a model representing the USD/HKD exchange rate as a stochastic process. This approach was first introduced for stock prices [6]. By the latter half of the $20^{\text {th }}$ century, the stochastic and unpredictable nature of price dynamics had been well established as the predominant viewpoint [7]. A review of the literature covering the developments during this period can be found on pages $37-39$ of Shiryaev [8]. There is overlap between the various statistical distributions proposed for stock prices and foreign exchange rates, for example, the Gaussian distribution which was used by Black and Scholes [9], stable distributions [10, 11, 12], the Student distribution [13,14], and distributions with time varying parameters [15]. Foreign exchange processes have also been characterized as autoregressive time series with conditional heteroscedasticity $(\mathrm{ARCH})[16]$ and self-similar fractals $[17,18]$ especially when high frequency intraday data is considered.

It appears that the existing models of stock prices and foreign exchange rates need to be reconsidered to take into account the boundedness of the USD/HKD exchange rate. A model for a theoretical exchange rate under a currency band has been suggested such that the dynamics are mean reverting [19]. In the framework of this model, this dynamic is justified by the economic belief of market participants that within this bound, a credible level for the exchange rate exists. A theoretical investigation of this model postulates that deviations from this credible level beyond the bounds will be expected to incur correction by external intervention thus setting up the mean reversion and boundedness property. To our knowledge, there has been only one investigation of the USD/HKD exchange rate after the separation of strong and weak side convertibility undertakings [20]. This investigation has concluded that, for this exchange rate, the hypothesis of a mean reversion property is to be rejected. This shows that the problems of modelling of this particular process are not completely solved yet.

We reconsider this issue of a dynamic for a bounded exchange rate, specifically the USD/HKD exchange rate and the problem of reproducing it from its dynamics. In another paper, we show evidence of fractal dynamics exhibited by this exchange rate [21]. However, we assume no such prior knowledge in this approach. To that end, we specifically use the fact that the USD/HKD exchange rate is bounded in the interval $7.75-7.85$ to suggest the following model: a discrete time model wherein the distribution of process increments is completely specified and conditional on the current exchange rate. For the purposes of simulation, a discrete time model is considered. We tested two discrete time Markov models: a 1D model and a 2D model. For both of these 
models, we consider:

(i) the state distribution resulting from the set of all transition probabilities after the state space discretization with arbitrarily small step size

(ii) the volatility of the exchange rate process

We found that a 1D model was sufficient to replicate the long term state distribution and the bounded dynamic of the process. On the other hand, extension to 2D appears to better capture the volatility and dynamics of the historical process. Note the concurrent achievement of these objectives appears to be a difficult task and has not been achieved in the case of a continuous time model [20]. It should also be noted that the approach of this paper is not without restrictions as it can only be applied if the range of the process considered is finite as is the case for the USD/HKD exchange rate as considered here.

We model separately the rate behaviour inside the band and in the neighbourhood of the boundaries, as in the framework of Nicolau [22]. In this setting, the local dynamics of the process inside the open interval between the boundaries can be treated independently of the dynamics when within the presence of the boundaries. This is different from the approach in the mean reverting model [19]. This also allows consideration of other behaviours for the boundaries such as repulsion, reflection or impedance. Taking into account what has been observed of the historical USD/HKD exchange rate so far, we also suggest that the boundary can act as an attractor for the process, resulting in the process 'sticking' to the boundary for some time once it approaches too closely.

The rest of the paper is organized as follows: Section 2 describes the distribution of the process and possible dynamics; Section 3 suggests a discrete time Markov model to reproduce these dynamics and compares the simulated process and volatility to their historical counterparts; Section 4 modifies the model to account for correlation between adjacent increments resulting in the 2D Markov model.

\section{Historical distribution and dynamics}

We begin by attempting to characterize the distribution of the USD/HKD exchange rate process. Exchange rate values at which trades have been executed are sampled at one minute intervals, excluding non-trading times. These values are generally contained within the interval [7.75, 7.85] and are discretized to 4 decimal places. A histogram of these values attained over the period from 2008 until 2012 is shown in Figure 2.1. While the entire range of permissible values [7.75, 7.85] for the USD/HKD exchange rate is displayed, activity is mostly confined to the lower half of this range. Note also the peak at the lower boundary indicating a high frequency of trades around that particular exchange value.

Figure 2.2 contains a similar depiction for each of the 5 years in the 2008 to 2012 interval. In each of the 5 years, the distribution of values once again remains mostly in the lower half of the interval $[7.75,7.85]$. When observed yearly, the distributions appear to follow one of two distinct patterns:

(i) high peak at the 7.75 boundary with a large positive skew, similar to that seen in the original 5 year histogram (Figure 2.1)

(ii) more symmetrically dispersed with lower peak(s) within the interval $[7.75,7.85]$ away from the boundary. 


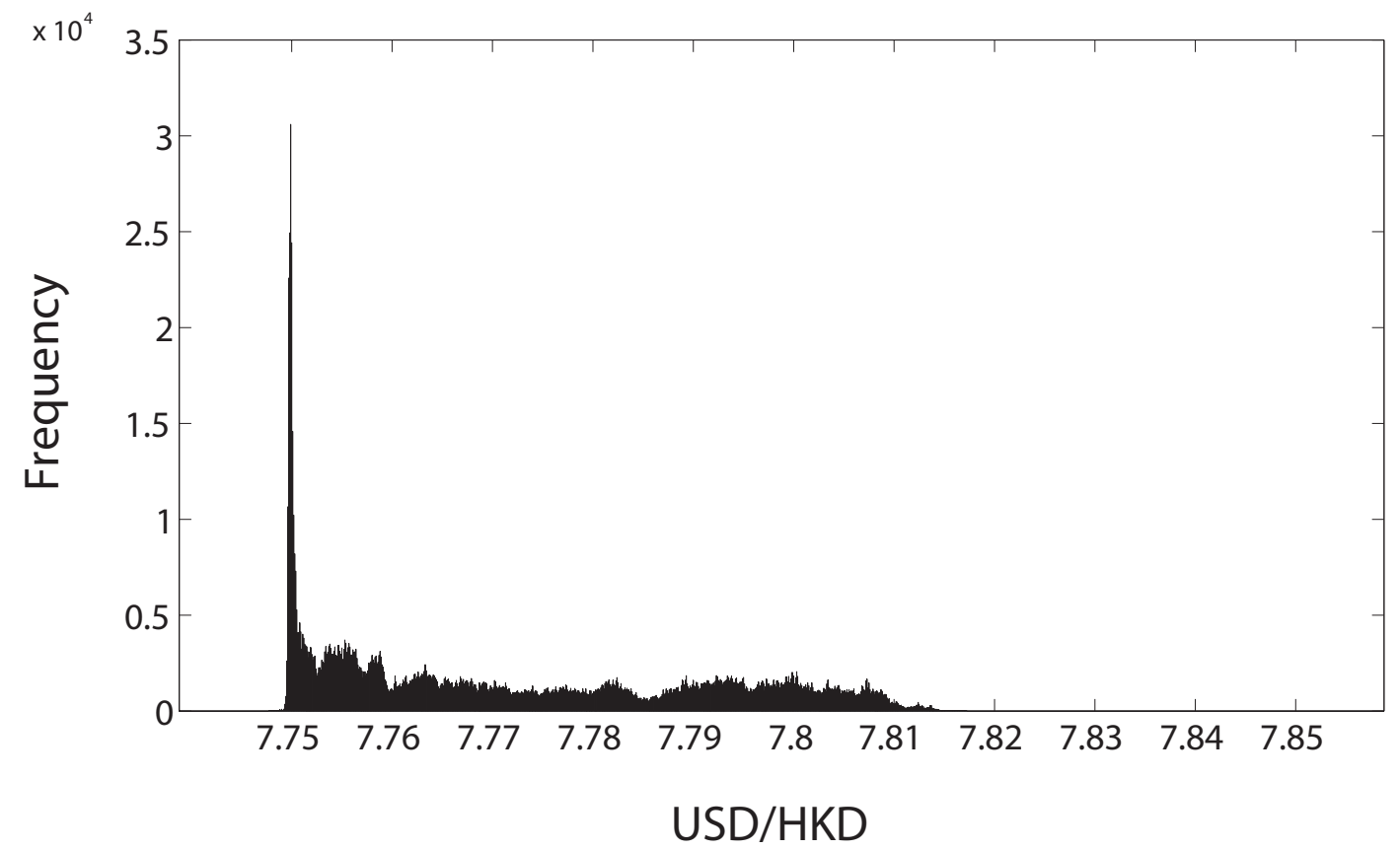

Figure 2.1: Frequency of USD/HKD exchange values from 2008 to 2012

Some years appear to be a mix between these two distributions. This would appear to suggest at least two distinct phases for this process:

(i) when the process is on or close to the 7.75 boundary and

(ii) when the process is further from the boundary from but still within the interval [7.75, 7.85]

which each histogram contains a mixture of as an annual snapshot. It is postulated that along the time axis, the process remains in one of these two phases with transitions occurring from one phase to the other. Over the entire 5 year history considered, this is masked by the high activity at the 7.75 boundary and is seen more clearly in the annual histograms which capture the process during and in between transitions.

\section{1D Markov model}

To investigate whether this exchange rate process can be reproduced, we propose a discrete time Markov model where increments in the process are assumed to be conditional on the process' current value. To specify these conditional increment distributions, the probability of events of the following type need to be determined:

$$
\mathrm{P}\left(X_{t+1}-X_{t}=\delta \mid X_{t}\right),
$$

where $X_{t}$ represents the value of the exchange rate process indexed by time $t$ where $t$ is the current time and $\delta$ is the value of the rate increment. Advantage is taken of the fact that the process is bounded within the interval $[7.75,7.85]$ as this restricts the possible values attainable by $X_{t}$ and hence the possible states which need to be conditioned upon. These probabilities are derived from frequentist estimates using historical exchange rate data which is discrete hence 


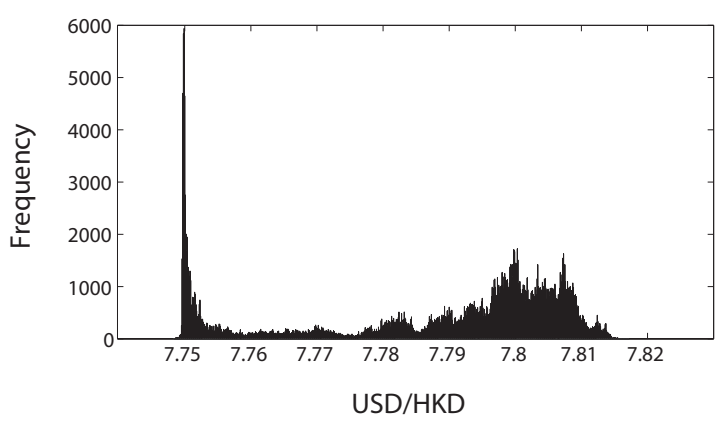

(a) 2008

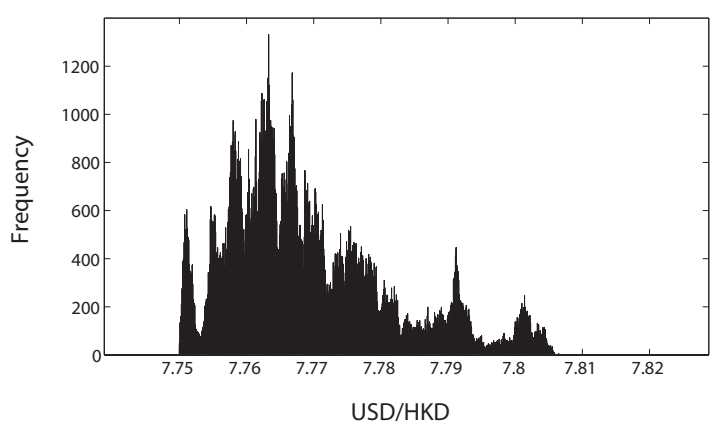

(c) 2010

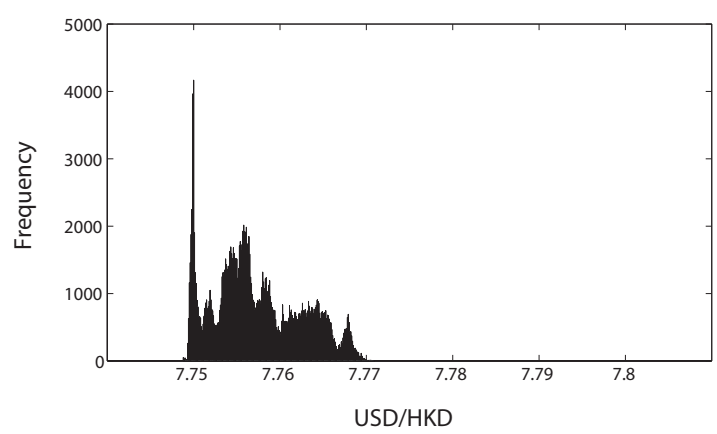

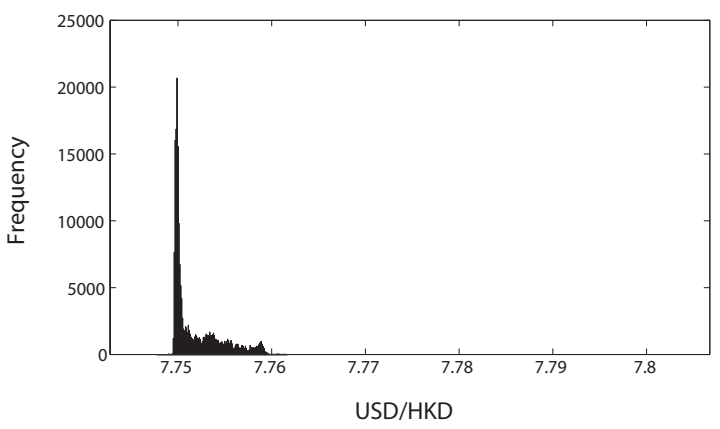

(b) 2009

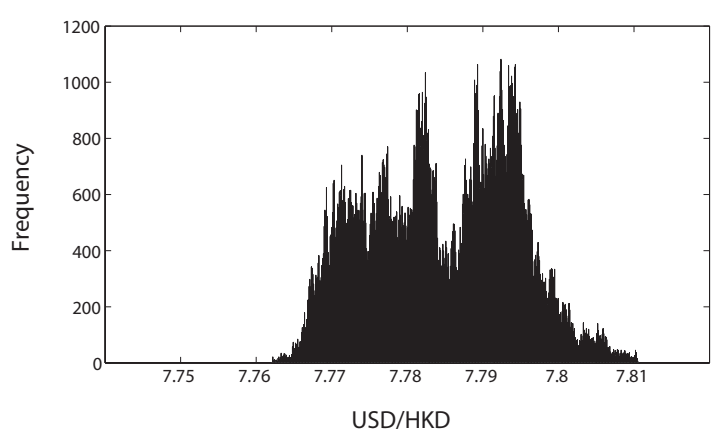

(d) 2011

(e) 2012

Figure 2.2: Frequencies of yearly USD/HKD exchange values

the specification of the conditional increment distribution as a discrete distribution. This does not however preclude the usage of continuous increment distributions in the model instead.

Traditionally, the increment between the logarithm of the process values is examined rather than the increment between the values directly. In this case, since the process takes only values that are numerically small and are bounded within a similarly small interval, the application of the logarithm is forgone. Increments in the process are calculated as $\left[X_{t+1}-X_{t}\right]$ and paired with the current value $X_{t}$. Histograms of the conditional distribution of increments in the process $\left[X_{t+1}-X_{t}\right]$ for each current value $X_{t}$ are collated to form a 3 dimensional surface as shown in Figure 3.3. This shows the distribution of increments with respect to the condition of current state $X_{t}$. A close-up is shown in Figure 3.4 where it can be more clearly seen that the conditional distribution of process increments are approximately symmetrically distributed and centrally clustered around mean 0 at most of the current values $X_{t}$ attained in the interval 


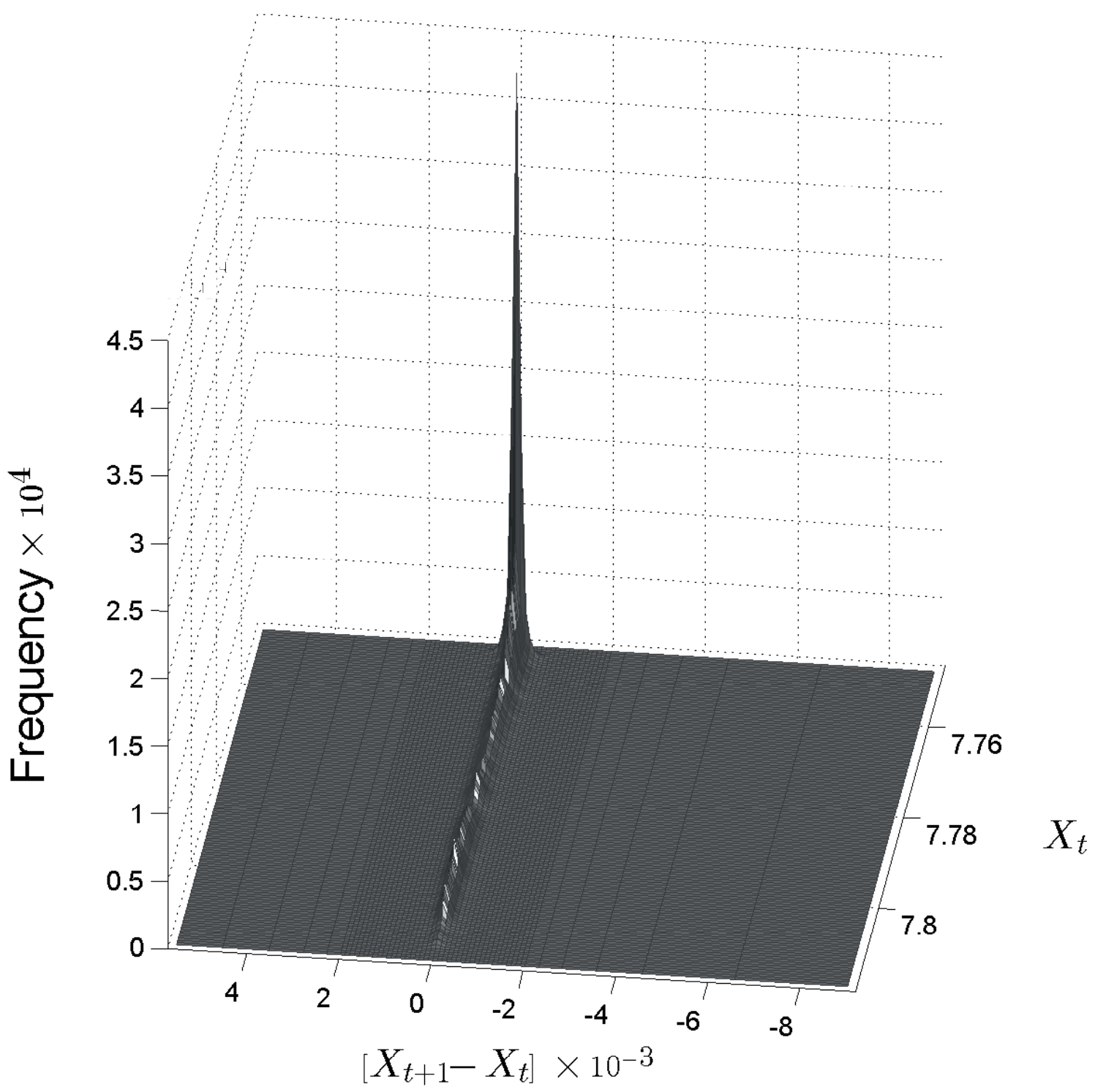

Figure 3.3: Frequency of increments conditional on current state of process

$[7.75,7.82]$ upon which the distributions are conditioned. Note that at the lower boundary of 7.75 there are a large number of increments associated with the values in the range [7.75, 7.76]. This is consistent with the histograms from Figure 2.1 and Figure 2.2 showing high activity at the lower boundary. There is comparatively less activity and hence there are fewer increments at values beyond the interval $[7.75,7.82]$. While this can be seen in Figure 3.4 for values of $X_{t}$ greater than 7.81 , this is obscured at the 7.75 boundary by the high peak in the interval $[7.75,7.76]$. 


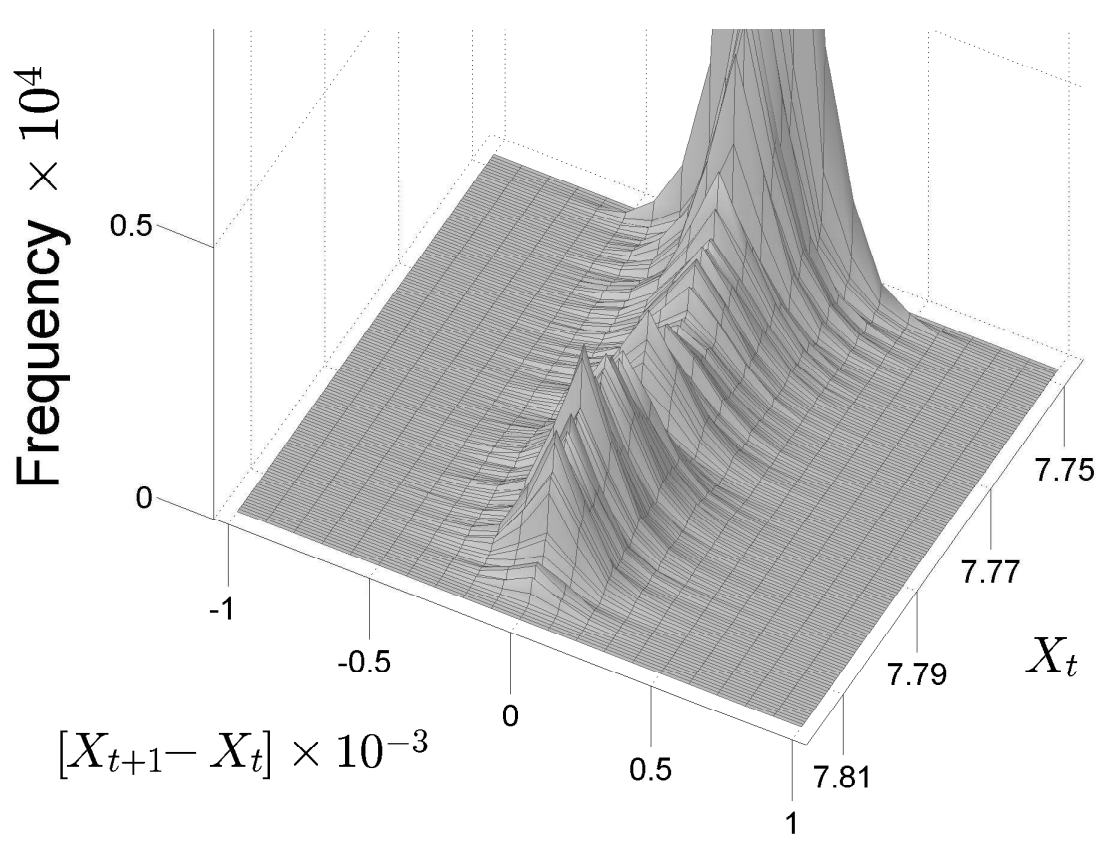

Figure 3.4: Frequency of increments conditional on current state of process (Close-up)

From these frequencies, the required probability estimates for the conditional increment distributions are obtained by normalising the frequencies at each current state $X_{t}$. This by definition preserves the structure of the distributions obtained from the historical data. To better show the distribution of increments at the extremities, the conditional mean (2) and variance $(3)$

$$
\begin{gathered}
\mathrm{E}\left(X_{t+1}-X_{t} \mid X_{t}\right), \\
\operatorname{Var}\left(X_{t+1}-X_{t} \mid X_{t}\right),
\end{gathered}
$$

of the distribution of increments $\left[X_{t+1}-X_{t}\right]$ are calculated

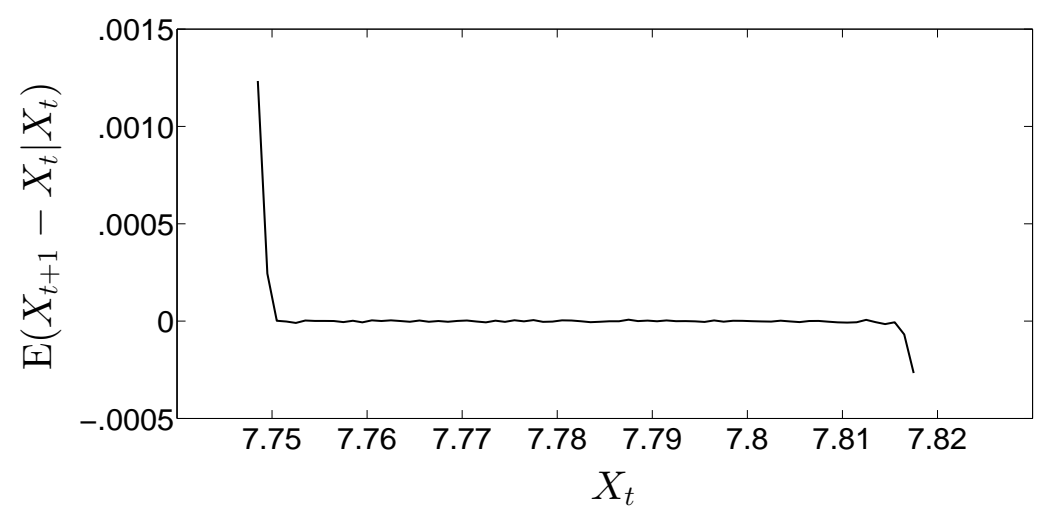

Figure 3.5: Mean of increments conditional on current value

in Figure 3.5 and Figure 3.6 respectively. For the values assumed by the process $X_{t}$ in the interval $[7.75,7.82]$ away from the end points, the distributions of increments have relatively constant variance and close to 0 mean. This would allow the dynamics of the process to conform 


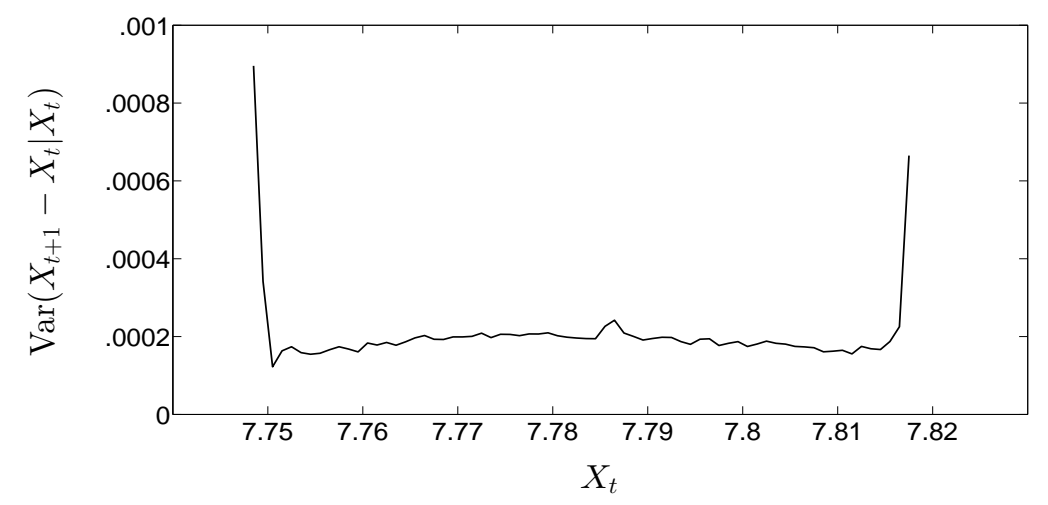

Figure 3.6: Variance of increments conditional on current value

to a random walk at such values of $X_{t}$. Of more interest however is the distribution at the end points of the interval $[7.75,7.82]$. At the lower boundary of 7.75 , the mean of the increment distribution becomes positive, indicating upward movement of the process when at these values. Similarly, at the upper interval end point of 7.82 , the mean becomes negative indicating downward movement. Inspection of the distributions at the upper and lower boundary reveal their supports to be transitioning to a more non-positive and non-negative nature respectively. Based on these characteristics, the upper boundary of 7.85 might be described as a repelling boundary and the lower boundary of 7.75 an impassable boundary with some degree of stickiness. An alternative characterization of the lower boundary might be a reflecting boundary with delay, such that the process remains on the boundary with probability one for some period of time.

\subsection{Comparison of historical process and simulated prices using the 1D model}

The conditional increment distribution required to completely specify the Markov model (1) is obtained by normalising the conditional frequency distribution from Section 3. This discrete time Markov model is then used to simulate the USD/HKD exchange rate process. By virtue of its construction, it is expected that over a sufficiently long time horizon, histograms of the simulated process will be virtually indistinguishable from the historical process. It appears that this is indeed the case therefore further analysis of the long term distribution of the simulated process becomes moot. Instead, we consider another important parameter used in the description of financial processes, that is the volatility.

By convention, the logarithm of returns (log returns) is used in the estimation of volatility. For the sake of simplicity, a static estimator of volatility, the standard deviation, is used. To facilitate comparison, the number of observations selected for the calculation is scaled to represent one year. This results in the traditional volatility estimate [23]

$$
\sigma^{2}=\frac{1}{\left(m-m_{0}\right) \delta} \sum_{k=m_{0}}^{m}\left(\log Z_{t_{k}}-\mu\right)^{2},
$$

over an interval $[t-\Delta t, t]$ with $t$ measured in years where $m_{0}$ and $m$ are the indices for initial time $t_{m_{0}}=t-\Delta t$ and final time $t_{m}=t$ respectively and $\delta=t_{k+1}-t_{k}$ is the sampling resolution which is the time difference between two adjacent time intervals. If observations taken are such that they span an interval of one year, $\left(m-m_{0}\right) \delta=1$ since $\Delta t=1$ and the number of intervals 
$\left(m-m_{0}\right)=n-1$ is related to the sampling resolution by $n-1=\frac{\Delta t}{\delta}$. Equation (4) simplifies to a sum of the entire year's values as the scaling fraction preceding the sum reduces to 1 . This is used to calculate the annual historical volatilities for each of the five years as tabulated in Table 1. Since the entire five year dataset is available, each of the annual volatilities are calculated from an entire year's worth of data without any additional scaling. We compare this to volatilities

\begin{tabular}{|l|c|c|c|c|c|}
\hline Year & 2008 & 2009 & 2010 & 2011 & 2012 \\
\hline$\sigma$ & 0.01277 & 0.00783 & 0.01123 & 0.01088 & 0.00892 \\
\hline
\end{tabular}

Table 1: Annual Historical Volatility (standard errors in the order of magnitude $10^{-7}$ )

obtained from simulations of the USD/HKD exchange rate process producing a year's worth of exchange rate values. Figure 3.7 shows the distribution of volatilities calculated from 100000 of these simulations. The volatilities for all five years could be reasonably contained within the

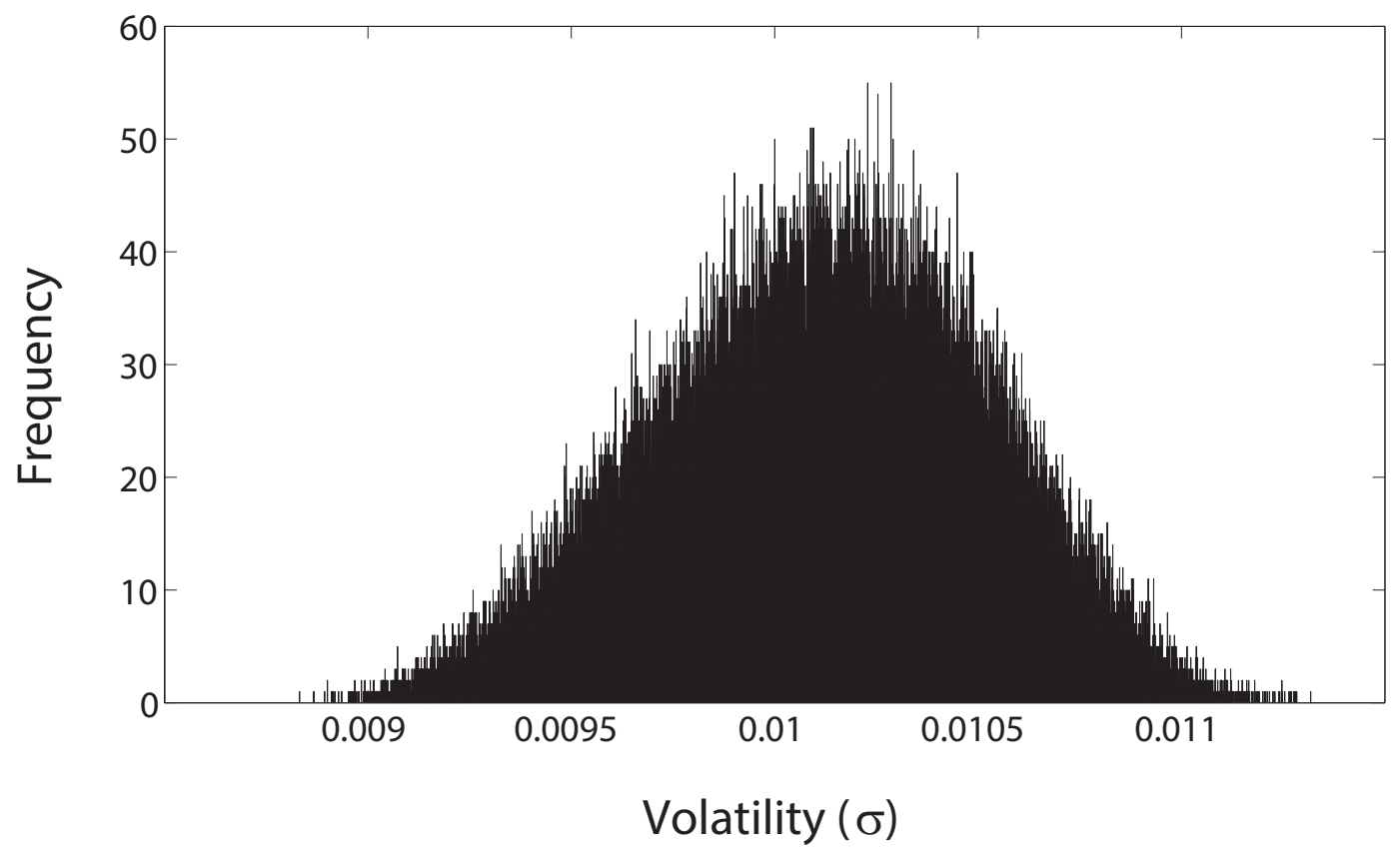

Figure 3.7: Frequency of volatilities from 1D Markov model simulations

support of this distribution though the more extreme volatilities could be cause for concern.

To further ascertain the suitability of the Markov model in reproducing the USD/HKD exchange rate process, we compare a 5 year simulation using the Markov model with the historical data over the 5 year interval from 2008 to 2012. Instead of analysing static distributions using histograms, the evolution of the exchange rate process through time is compared. Figure 3.8 shows the historical exchange rate process over the years from 2008 to 2012 . Compare this with a simulation as shown in Figure 3.9 over a five year duration using the previously mentioned Markov model. The overall trends of the simulated process do exhibit some necessary characteristics such as the maintenance of the lower boundary at 7.75 as well as the tendency to remain in that region for more time as compared to other possible levels attainable by the process. Closer inspection of short stretches of both processes however reveal that the simulated process fluctuates more. This is most clearly seen when the processes remain at the lower boundary as 


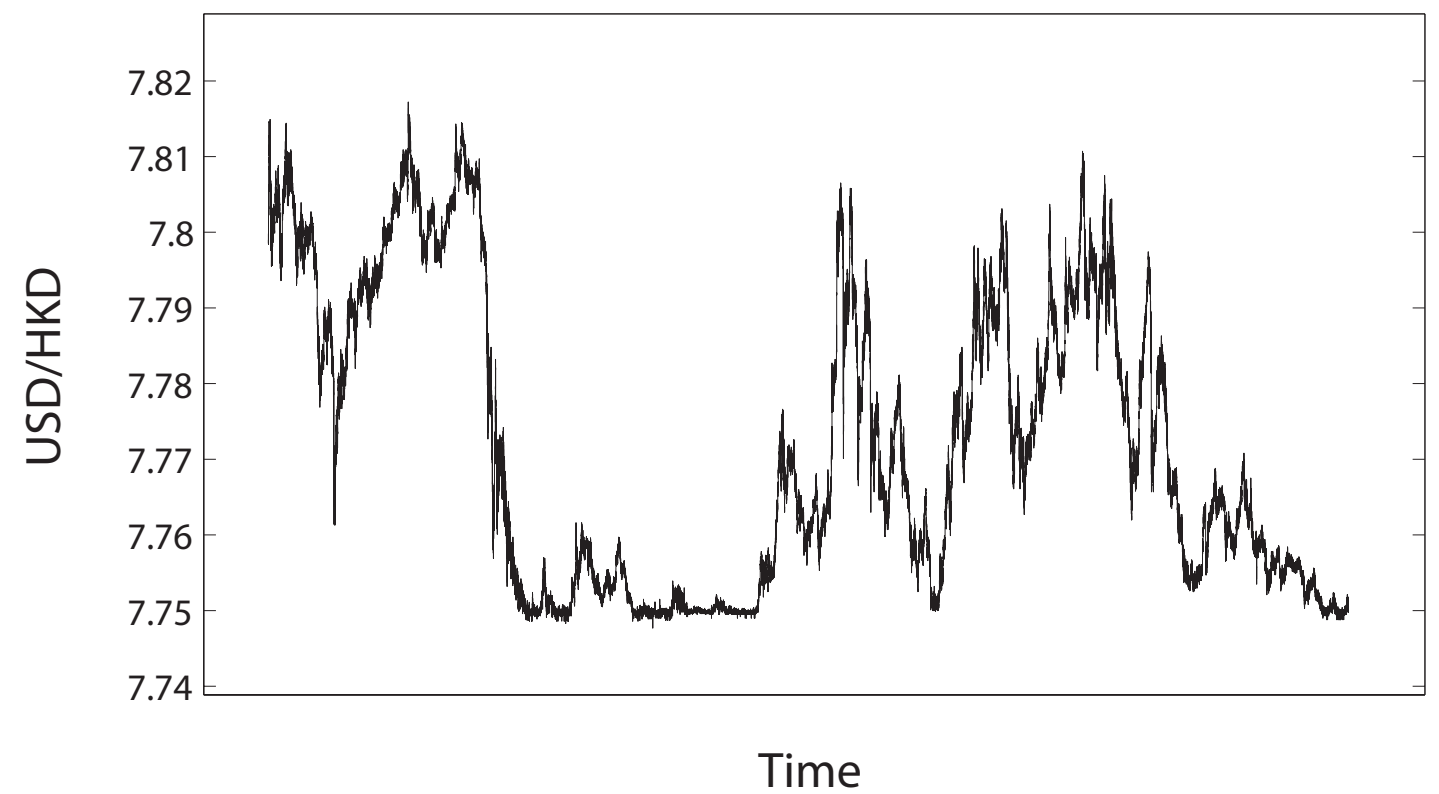

Figure 3.8: 5 year historical evolution of USD/HKD exchange rate

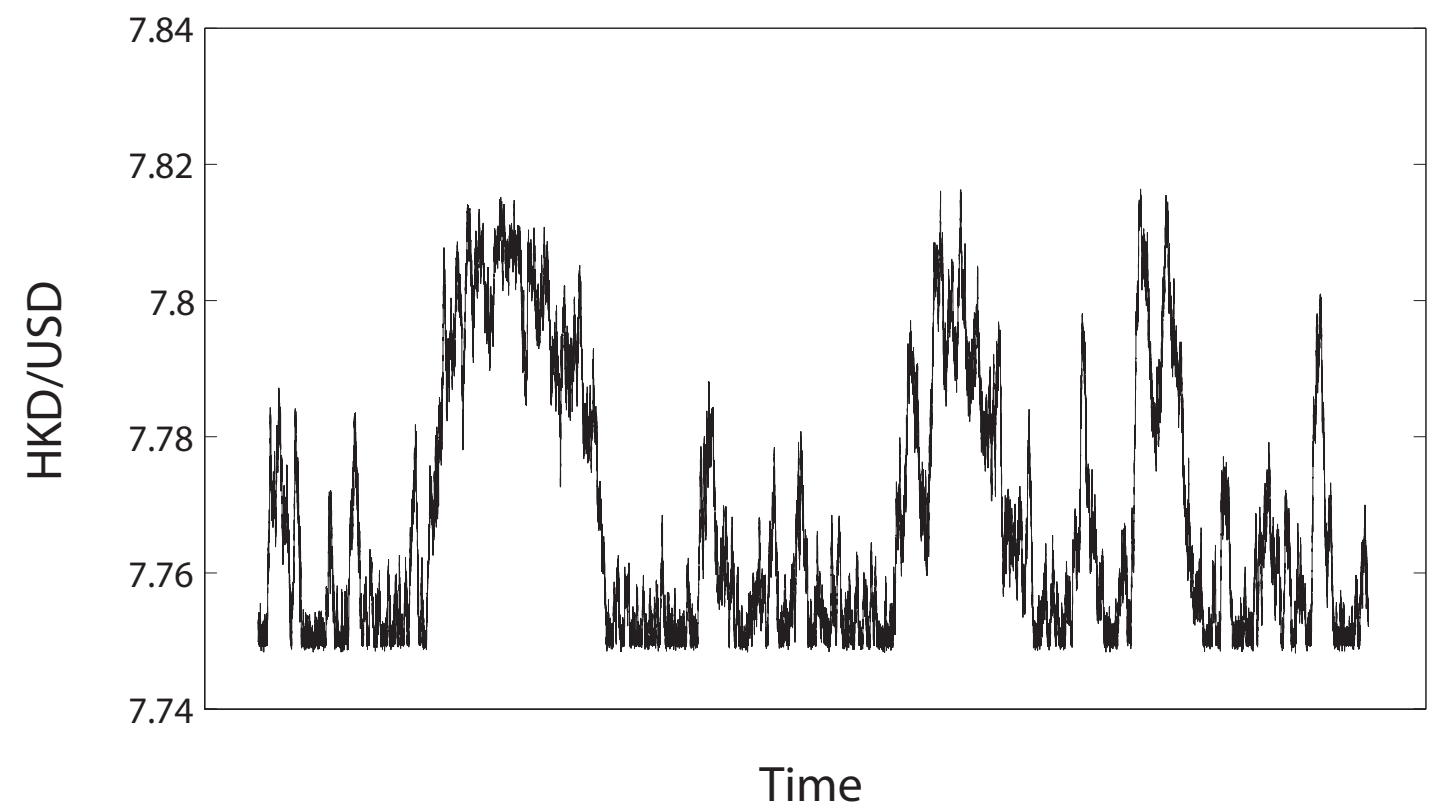

Figure 3.9: 5 Year simulated evolution of USD/HKD exchange rate process using 1D Markov model

the more frequent departure by the simulated process from the lower boundary compared to the historical process.

The effects of this apparently greater fluctuation would be an increase in the volatility of the 
simulated process in addition to the visual differences seen in the plots of the processes against time. This fluctuation can be attributed to the assumption of independence of increments in the Markov model. High frequency intraday foreign exchange data displays a significantly negative first lag autocorrelation in their returns, unlike the independent returns observed at a coarser time resolution [24]. Returns being simply the increments in the logarithm of the process, it is not inconceivable that the increments of the process directly also demonstrates this as well.

\section{2D Markov model}

Consider instead conditional probabilities for the process increments

$$
Y_{t}=X_{t+1}-X_{t}
$$

and to construct a model for a 2D Markov process using $\left(X_{t}, Y_{t}\right)$. To ascertain the independence of increments $Y_{t}$, we estimated the time lagged correlation or autocorrelation for the historical USD/HKD exchange rate dataset. While the increment $Y_{t-1}$ appears to depend on $X_{t}$ as seen in (5) for $Y_{t-1}$, there should be no direct correlation between $X_{t}$ and $Y_{t-1}$ as arrival at a particular $X_{t}$ can be achieved via any number of different increments $Y_{t-1}$ depending on the value of $X_{t-1}$. The autocorrelation at a time lag $k$ is

$$
\gamma_{k}=\frac{\operatorname{Cov}\left(Y_{t}, Y_{t-k}\right)}{\operatorname{Var} Y_{t}}
$$

and Figure 4.10 shows the autocorrelation of $Y_{t}$ for up to 25 time lags. Only lags $k=1,2$

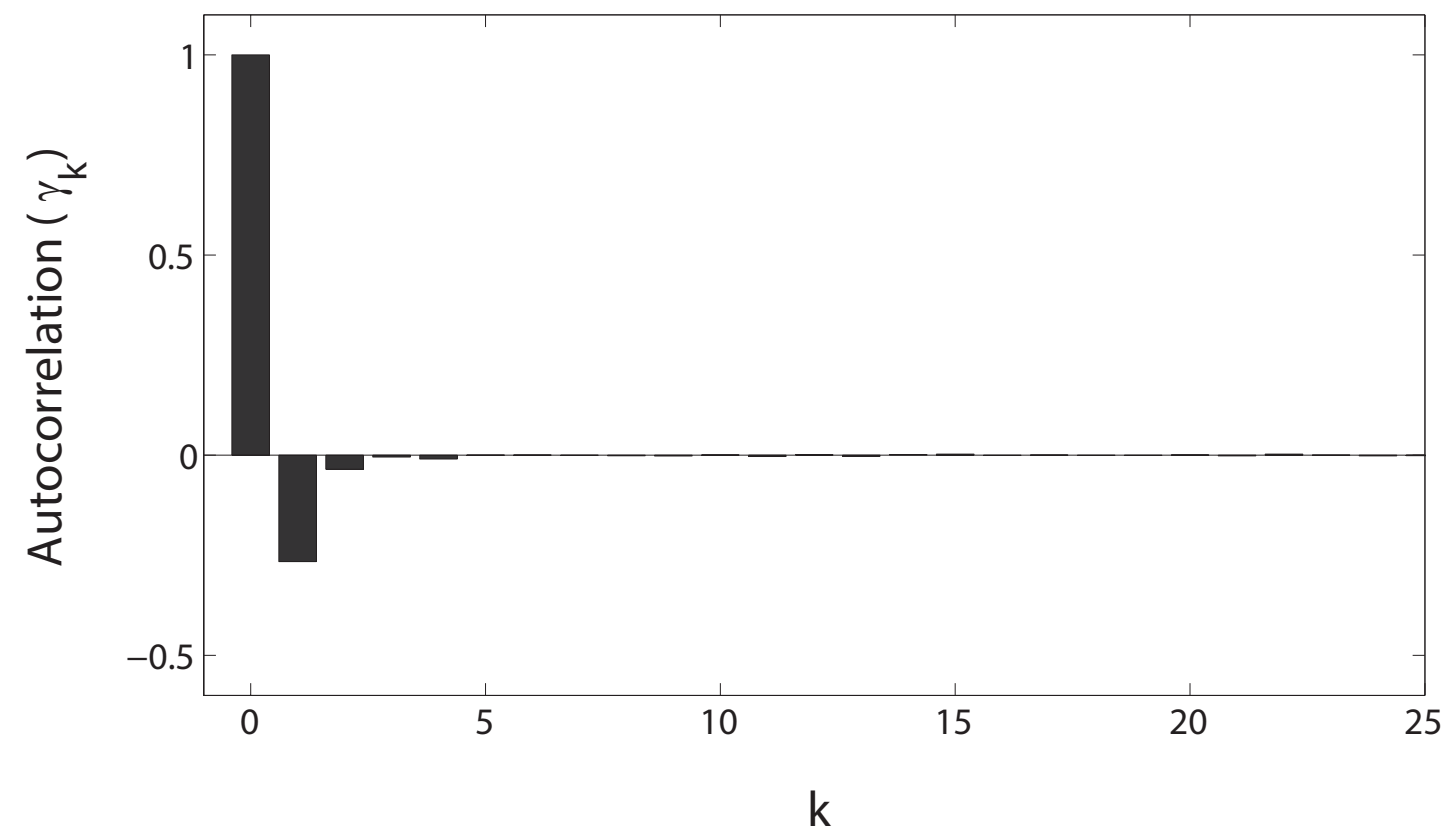

Figure 4.10: Autocorrelation plot of increment $Y_{t}$ up to lag 25

show any appreciable correlation. Table 2 shows the autocorrelations for the first 5 lags. The remaining autocorrelations do not exceed 0.005 in magnitude. The first lag autocorrelation is 


\begin{tabular}{|l|c|c|c|c|c|}
\hline$k(\mathrm{Lag})$ & 1 & 2 & 3 & 4 & 5 \\
\hline$\gamma_{k}$ & -0.2658 & -0.0349 & -0.0047 & -0.0094 & 0.0001 \\
\hline
\end{tabular}

Table 2: Autocorrelation of increment $Y_{t}$ for lags 1 to 5 (standard error: $9.707 \times 10^{-4}$ )

sufficiently large to refute any notion of the possible independence of two immediately adjacent increments. Taking this into account, the Markov model (1) is extended by incorporating this dependency as follows:

$$
\mathrm{P}\left(X_{t+1}-X_{t}=\delta \mid X_{t}, X_{t}-X_{t-1}\right)
$$

or, more concisely,

$$
\mathrm{P}\left(X_{t+1}-X_{t}=\delta \mid X_{t}-X_{t-1}\right) .
$$

While it is no longer strictly Markov in $X_{t}$ it may still be considered Markov in $Y_{t}$ as (6) can be rewritten as

$$
\mathrm{P}\left(Y_{t}=\delta \mid Y_{t-1}\right) \text {. }
$$

The model in (7) shall be referred to as the 2D Markov model for the process $\left(X_{t}, Y_{t}\right)$, where $Y_{t-1}=X_{t}-X_{t-1}$.

As before, a conditional frequency distribution is obtained for the 2D Markov model and normalized to produce the probabilities for the conditional increment distribution. As the frequencies are being conditioned on the two states $X_{t}$ and $X_{t-1}$ required to obtain $Y_{t-1}$, a graphical representation is not feasible as it would require a 4 dimensional display, the additional dimension coming from the added state to condition upon. This model is then used to simulate the exchange rate process and compare the volatility produced by this model to that obtained from the historical process, similarly to what was done for the Markov model in Section 3.1. The volatility distribution as shown in Figure 4.11 is obtained from 150000 simulations of the 2D Markov model, each over a time period representing one year. A sample trajectory of the process over a 5 year interval is shown in Figure 4.12. The introduction of this dependency in the increments to the model has had two notable effects. With regards to the volatility of the model, Figure 4.11 shows that the support of the volatility distribution has been extended to include lower volatilities. When compared to the historical volatilities in Table 1, this support does not extend too far beyond what is historically observed. Comparing the simulated 5 year process by the model to the previous, the moment to moment fluctuation is reduced but the overarching trends and boundary behaviour remain the same. Note that this results in a greater tendency for the process to remain at the lower boundary without departing as frequently as compared to the previous model.

\section{Distributions of the 2D Markov model}

The long term distribution of the 2D Markov model should approach the historical distribution by virtue of the model's construction. This, in and of itself, should be no surprise; what is of interest however, is the distribution at intermediate time intervals and the stability of this long term distribution. Instead of simulating a realization of values of $X_{t}$ as done previously, the exact one-step distribution as contained within the model is used to derive the distribution of values for subsequent steps. In other words, using the known probabilities from the model in

$$
\mathrm{P}\left(Y_{t}=\delta \mid Y_{t-1}\right)=\mathrm{P}\left(X_{t+1}=X_{t}+\delta \mid X_{t}, X_{t-1}\right),
$$




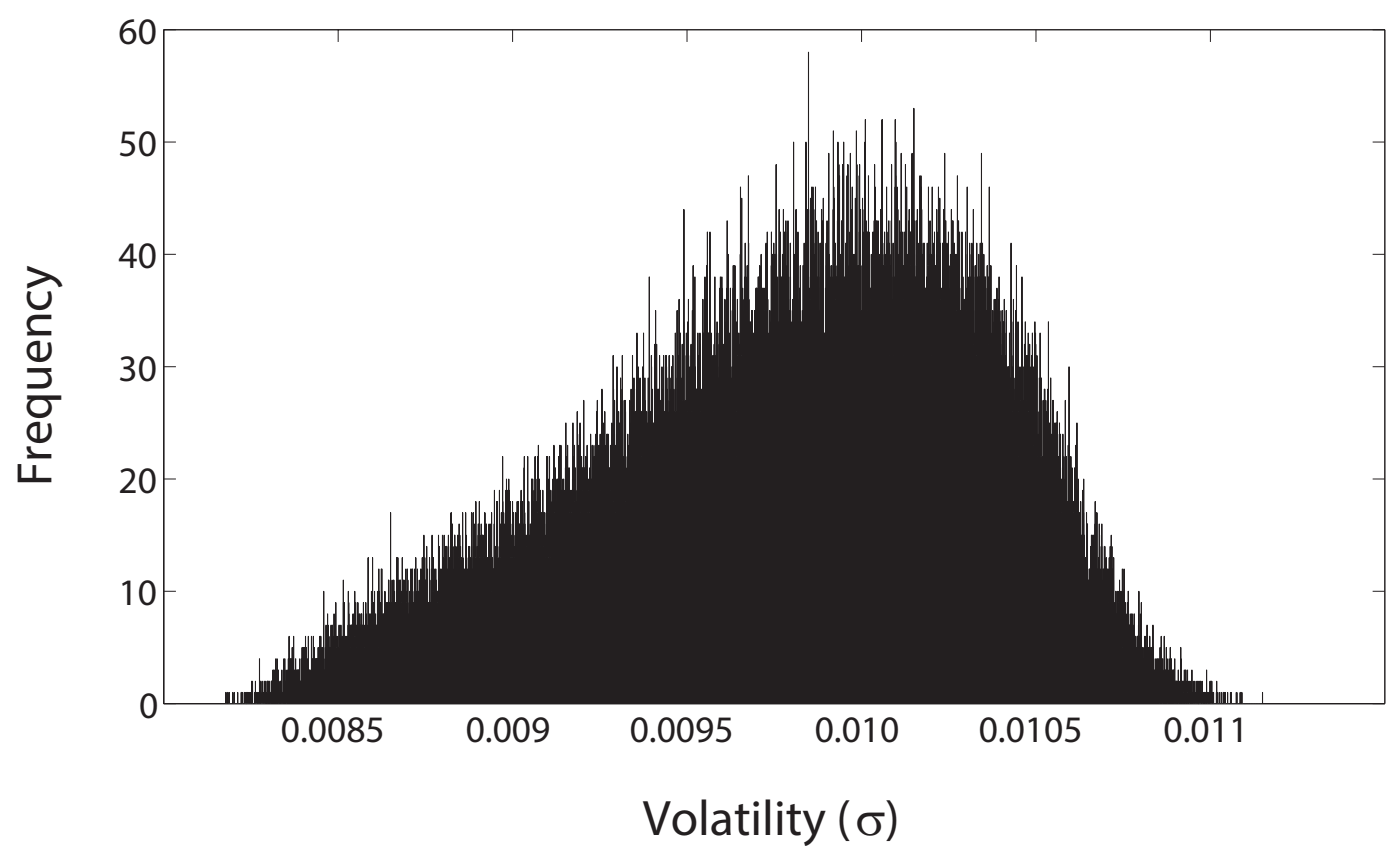

Figure 4.11: Frequency of volatilities from 2D Markov model simulations

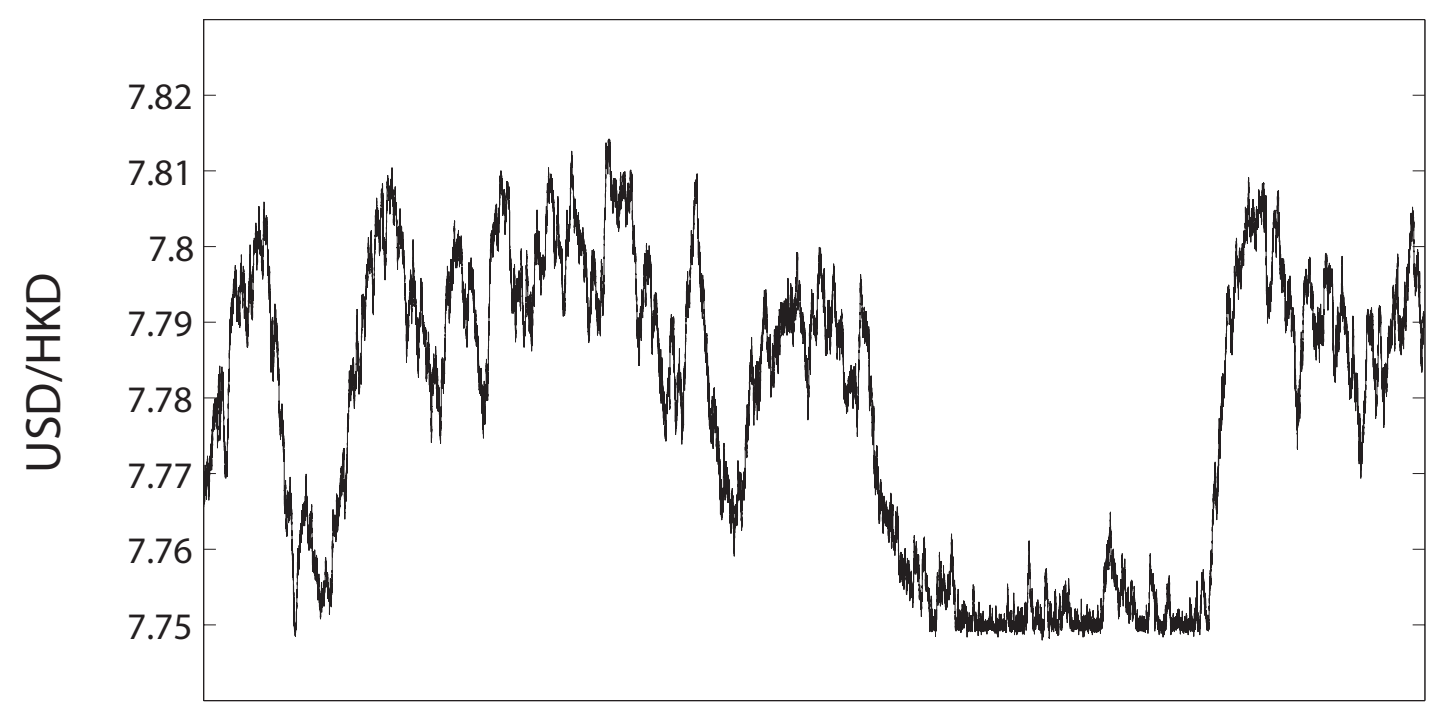

Time

Figure 4.12: 5 Year Simulated evolution of USD/HKD exchange rate process using 2D Markov model the probabilities

$$
\mathrm{P}\left(X_{t+k}=x \mid X_{t}, X_{t-1}\right), \quad x=7.75+i \times 10^{-4}, \quad \begin{aligned}
& i \in\{0,1, \ldots, 1000\} \\
& k \in \mathbb{N}
\end{aligned},
$$

can be derived to obtain the distribution of $X_{t+k}$. 
The general finite state Markov model with $n$ states can be represented using $n$-dimensional vectors $\mathbf{v}_{t}$ and matrices $\mathbf{M}$ with values in $\mathbb{R}^{n \times n}$ as follows

$$
\left(\mathbf{v}_{t+1}\right)^{\mathrm{T}}=\left(\mathbf{v}_{t}\right)^{\mathrm{T}} \mathbf{M}
$$

Here the $i^{\text {th }}$ element of the vector $\mathbf{v}$ is the probability associated with the $i^{\text {th }}$ state out of the possible $n$ states; the $i, j^{\text {th }}$ element ( $i^{\text {th }}$ row, $j^{\text {th }}$ column) of the matrix $\mathbf{M}$ is the probability of transitioning from the $i^{\text {th }}$ state to the $j^{\text {th }}$ state in one time step. The vector $\mathbf{v}_{t}$ represents the distribution of the $n$ states at time $t$. Each row of the matrix $\mathbf{M}$ represents a conditional one-step distribution. In terms of the individual components, (10) can be written as

$$
\left(\mathrm{v}_{t+1}\right)_{j}=\sum_{i=1}^{n}\left(\mathrm{v}_{t}\right)_{i}(\mathrm{M})_{i, j} \quad, \quad \forall j \in\{1, \ldots, n\} .
$$

To obtain the distribution of $X_{t}$ which is an underlying component of the Markov process $\left(X_{t}, Y_{t}\right)$, it is possible to adapt (9) to the machinery of Markov models as in (10) by considering ordered pairs such as $\left(X_{t}, X_{t-1}\right)$ and its subsequent step $\left(X_{t+1}, X_{t}\right)$ as the new states of another model, this results in a Markov model with $n^{2}$ states and a sparse $n^{2} \times n^{2}$ transition matrix. The number of new states, as well as transition matrix sparsity, increases exponentially as determined by the dependency on prior times, for example, in extending the dependency by another time period, ordered triples are considered resulting in $n^{3}$ new states and a $n^{3} \times n^{3}$ transition matrix. When dealing with a dependency of $d$ prior states, the transition matrix increases to dimension $\left(n^{d}\right)^{2}$. This quickly leads to unwieldy sparse transition matrices for large $d$ or even with small $d$ with large enough $n$.

To circumvent this difficulty, consider (11) as a binary operation between a first order and second order tensor yielding a first order tensor. Instead of extending this to higher order dependencies by considering ordered sequences of consecutive states, the corresponding transition step can instead be represented as a binary operation between higher order tensors. For instance, instead of extending (9) by considering ordered state pairs, the transition step can be represented as a binary operation between a second order tensor $\mathbf{v}_{t} \in \mathbb{R}^{n^{2}}$ and a fourth order tensor $\mathbf{M} \in \mathbb{R}^{n^{4}}$ yielding a second order tensor $\mathbf{v}_{t+1}$. The fourth order tensor represents the general multilinear transformation on the space of second order tensors and can be represented component-wise as

$$
\left(\mathrm{v}_{t+1}\right)_{i_{3}, i_{4}}=\sum_{i_{1}=1}^{n} \sum_{i_{2}=1}^{n}\left(\mathrm{v}_{t}\right)_{i_{1}, i_{2}}(\mathrm{M})_{i_{1}, i_{2}, i_{3}, i_{4}} \quad, \quad \forall i_{3}, i_{4} \in\{1, \ldots, n\},
$$

noting also that the dimensions of the tensors are the same as that of the elements derived from the extension using ordered state pairs. More generally, a dependency on $d$ prior states requires a $d^{\text {th }}$ order tensor where the $i_{1}, i_{2}, \ldots, i_{d}^{\text {th }}$ element represents the probability associated with the consecutive sequence of states

$$
\left\{\begin{aligned}
X_{t-d+1} & =f\left(i_{1}\right), \\
X_{t-d+2} & =f\left(i_{2}\right), \\
& \vdots \\
X_{t-1} & =f\left(i_{d-1}\right), \\
X_{t} & =f\left(i_{d}\right)
\end{aligned}\right\},
$$


and $f$ is the one-to-one mapping between the state values and their numbering. The analogue of the transition matrix is a $(2 d)^{\text {th }}$ order tensor representing the general multilinear transformation on the space of $d^{\text {th }}$ order tensors where the $i_{1}, i_{2}, \ldots, i_{2 d}{ }^{\text {th }}$ element represents the transition probability from the sequence of states

$$
\left\{X_{t-d+1}=f\left(i_{1}\right), X_{t-d+2}=f\left(i_{2}\right), \ldots, X_{t-1}=f\left(i_{d-1}\right), X_{t}=f\left(i_{d}\right)\right\}
$$

to the sequence

$$
\left\{X_{t-d+2}=f\left(i_{d+1}\right), X_{t-d+3}=f\left(i_{d+2}\right), \ldots, X_{t}=f\left(i_{2 d-1}\right), X_{t+1}=f\left(i_{2 d}\right)\right\} .
$$

For times $t-d+2$ to $t$, there is an overlap of states which need to correspond in order for the transition probability to be non-zero; the transition probability must be zero unless the following holds:

$$
X_{t-d+s}=f\left(i_{s}\right)=f\left(i_{d+s-1}\right) \quad, \quad \forall s \in\{2,3, \ldots, d\} .
$$

For the model (8), $d=2$ and it can be concluded that unless $i_{2}=i_{3}$

$$
(\mathrm{M})_{i_{1}, i_{2}, i_{3}, i_{4}}=0 \quad, \quad \forall i_{2} \neq i_{3}
$$

and because of this, one dimension of the fourth order tensor $\mathbf{M}$ is redundant and a third order tensor is sufficient to represent the necessary transition probabilities. In general, $d-1$ dimensions are redundant and a $(d+1)^{\text {th }}$ order tensor is sufficient to represent all possible transition probabilities. The summation over the indices is thus reduced to cover only the first index. The model (8) can then be represented using a second order tensor $\mathbf{v}_{t}$ containing the distribution of state pairs $\left(X_{t}, X_{t-1}\right)$ and a third order tensor $\mathbf{M}$ containing the necessary transition probabilities. The operation between the second order tensor and the third order tensor representing the one step time transition is shown in terms of individual components as follows

$$
\left(\mathrm{v}_{t+1}\right)_{j, k}=\sum_{i=1}^{n}\left(\mathrm{v}_{t}\right)_{i, j}(\mathrm{M})_{i, j, k} \quad, \quad \forall j, k \in\{1, \ldots, n\},
$$

The distribution of $X_{t}$ is obtained from the tensor $\mathbf{v}_{t}$ by summing the components along each dimension except the last resulting in a vector containing probability of each state at time $t$ regardless of the configuration of preceding states. For the case when $d=2$, this can be represented component-wise as

$$
\left(\mathrm{v}_{t}\right)_{j}=\sum_{i=1}^{n}\left(\mathrm{v}_{t}\right)_{i, j}
$$

As an illustration of significance of this reduction in dimensionality, in this study where $n \approx 700$ and $d=2$, the average office desktop computer can support a third order tensor of dimension $700^{3}$ but is unable to contain a matrix of dimension $700^{2} \times 700^{2}$.

Using this machinery, the Figures 5.13, 5.14 and 5.15 show snapshots of the evolution of the distribution of $X_{t}$ at several different times $t$ starting from 3 different sets of initial state pairs where $X_{0}$ is taken to be equal to $X_{1}$ for convenience. One year in real time is is approximately 200000 time steps. In Figure 5.14, the drift from the initial values can be seen as a widening of the distribution and flattening of the peak. As the distribution drifts towards the lower boundary at 7.75 , there is an accumulation of possible states at the lower boundary as evidenced by the new peak forming in the distribution. The distribution does not drift beyond the lower boundary and 

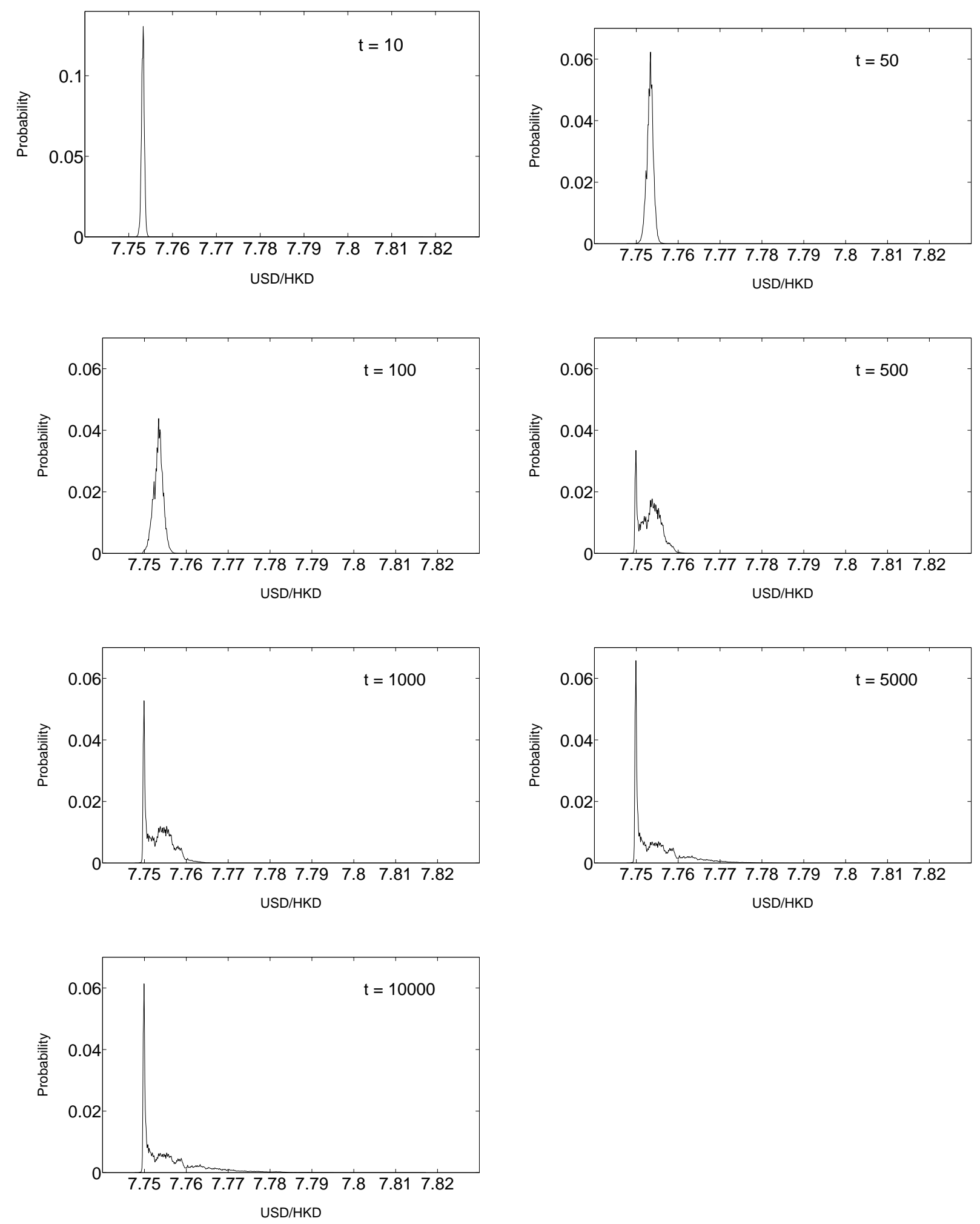

Figure 5.13: Evolution of $\mathbf{v}_{t}$ where $\left(\mathrm{v}_{t}\right)_{i}=\mathrm{P}\left(X_{t}=x_{i} \mid X_{0}=X_{1}=7.7532\right)$ 

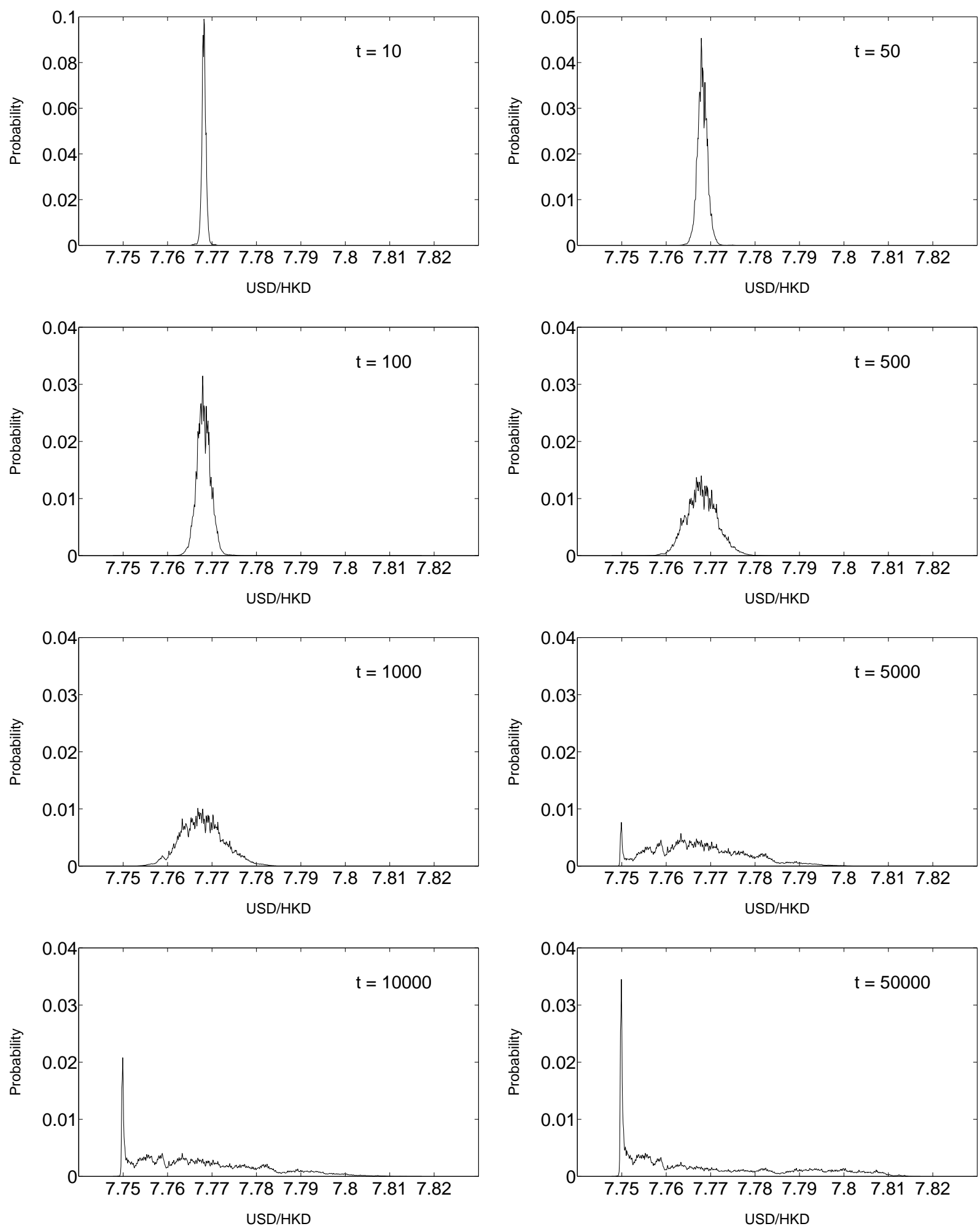

Figure 5.14: Evolution of $\mathbf{v}_{t}$ where $\left(\mathrm{v}_{t}\right)_{i}=\mathrm{P}\left(X_{t}=x_{i} \mid X_{0}=X_{1}=7.7682\right)$ 

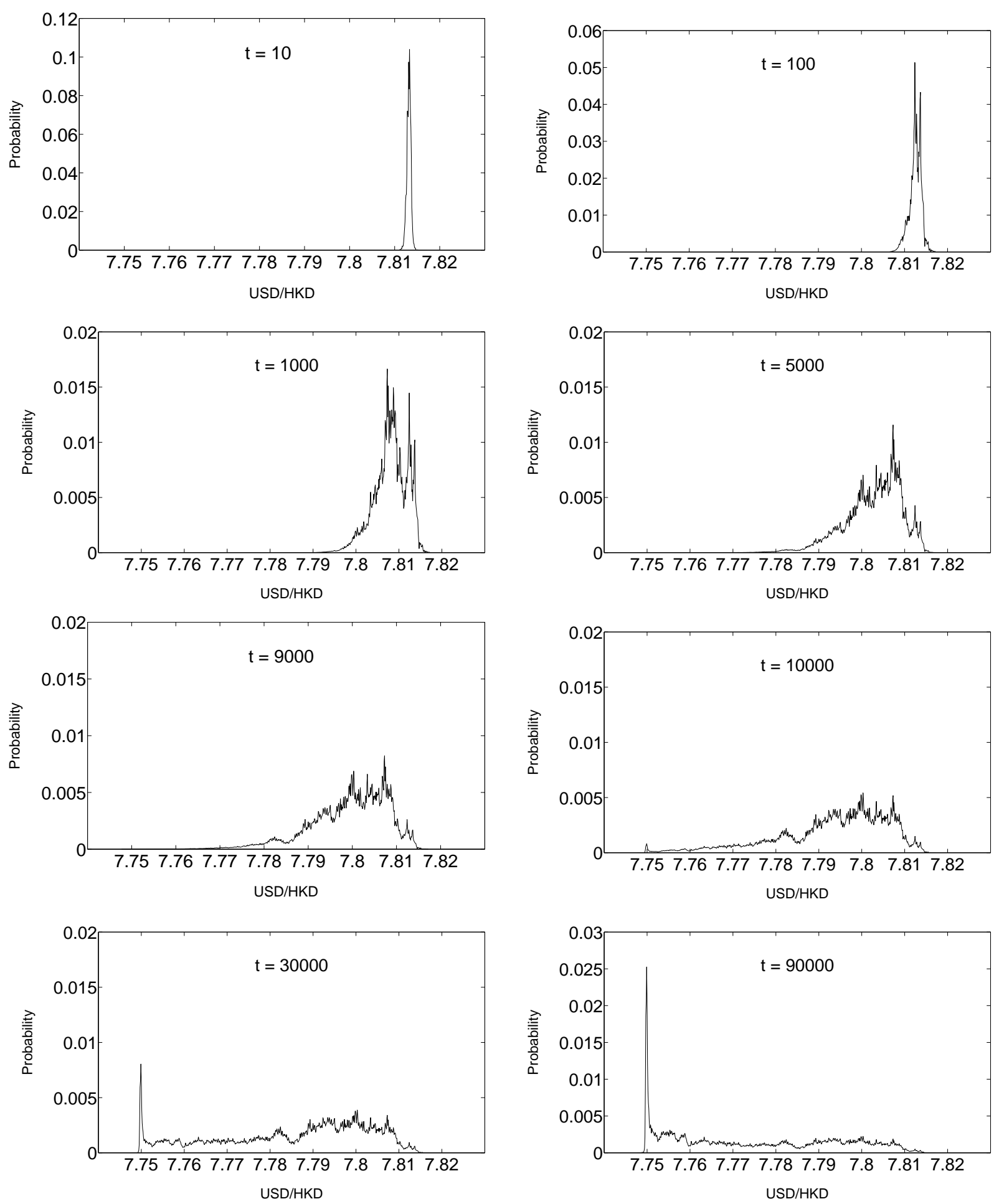

Figure 5.15: Evolution of $\mathbf{v}_{t}$ where $\left(\mathrm{v}_{t}\right)_{i}=\mathrm{P}\left(X_{t}=x_{i} \mid X_{0}=X_{1}=7.8132\right)$ 
does not realize any proximity with the upper boundary. These characteristics are consistent with what has been observed in the historical process as discussed previously.

Figure 5.13 shows a similar behaviour to that seen in Figure 5.14, the difference being that the starting values are closer to the lower boundary. This results in a quicker approach to the lower a boundary and subsequent formation of the resultant lower boundary peak. Both Figure 5.13 and Figure 5.14 eventually approach the same distribution which does not exhibit any further changes beyond a certain point in time, though this is not necessarily achieved at the same times. Figure 5.15 has a starting value beyond the mid-point of 7.80 and shows a slower spread of the distribution compared to the previous two cases. Given enough time, it will achieve the same distribution. For models based on historical data such as this, the issue of finding such a time invariant distribution and its dependence, if any, on the starting states, is thus motivated.

A time invariant distribution is a distribution vector $\mathbf{v}_{t} \in \mathbb{R}^{n}$ for an $\mathbf{M} \in \mathbb{R}^{n \times n}$ which satisfies the condition

$$
\left(\mathbf{v}_{t}\right)^{\mathrm{T}}=\left(\mathbf{v}_{t+1}\right)^{\mathrm{T}}=\left(\mathbf{v}_{t}\right)^{\mathrm{T}} \mathbf{M},
$$

and is termed a stationary distribution. An analogous definition holds for the general distribution tensor $\mathbf{v} \in \mathbb{R}^{n^{d}}$ with one step time transition as defined previously. For the case of model (8), the analogous representation in components is

$$
\exists t:\left(\mathrm{v}_{t}\right)_{j, k}=\sum_{i=1}^{n}\left(\mathrm{v}_{t}\right)_{i, j}(\mathrm{M})_{i, j, k} \quad, \quad \forall j, k \in\{1, \ldots, n\},
$$

If the same unique stationary distribution is achieved by long run transitions from any set of starting states, the model is termed ergodic. For a finite state model such as this, irreducibility of the states and a single aperiodic state is sufficient to ensure ergodicity [25].

(i) To ensure irreducibility, only the first and final state sets of the historical process need to be checked for inward and outward transitions respectively. All other state sets in the model are obtained from the intermediate states of the historical process and are therefore assured to belong to a single communicating class by virtue of these states forming a communicating chain. Irreducibility thus ensures that any state set in the model is eventually accessible from any other state set.

(ii) An aperiodic state is one in which

$$
\exists i_{1}=i_{2}=\cdots=i_{d+1}:(\mathrm{M})_{i_{1}, i_{2}, \ldots, i_{d+1}}>0, \quad \begin{aligned}
i_{r} & \in\{1, \ldots, n\} \\
r & \in\{1, \ldots, d+1\}
\end{aligned},
$$

for the general case of a dependency on $d$ prior states. The model (8) simply requires the checking of

$$
\exists i \in\{1, \ldots, n\}: \quad(\mathrm{M})_{i, i, i}>0
$$

that is the existence of at least one aperiodic state.

These two conditions are easy enough to ascertain and do hold for the model in (8). 


\section{Discussion and future research}

We have started by considering a 1D Markov model and extended to an improved 2D Markov model for the bounded exchange rate dynamic. The 2D Markov process appears to be a better match for the historical exchange rate process in terms of its dynamics and volatility. Since all available prices from the period considered were used, this did not lead to any information loss. Also, since we have used one of the higher recorded data frequencies, there is no immediate need to move to a continuous model capable of accommodating the data of an arbitrarily high frequency. Therefore, the choice of a discrete time model is justified from the practical point of view. Admittedly, continuous time models are more attractive for analytical methods since they allow the representation of prices at any frequency via equations with a small number of parameters.

There are additional challenges for the choice of continuous time models for bounded processes similar to USD/HKD exchange rate. The main problem is to take into account the boundedness. As was mentioned previously, there is evidence to support the characterization of the dynamics of the USD/HKD exchange rate as a diffusion Itô process when in between the boundaries. The mean reverting forces can be modelled via choice of the drift coefficient. The boundedness can be modelled via the choice of the drift and diffusion coefficients vanishing on the boundary such as in the model introduced by Hui and Fong [20]. This continuous model satisfies the conditions of boundedness but it does not allow the process to actually reach the boundary. This is acceptable when the boundary appears to repel the process as seen with the upper boundary. However, this model does not allow replication of the situation where the process remains on the boundary for a period of time, which appears to be typical for the lower boundary of the USD/HKD exchange.

This feature was captured by the discrete time model introduced in this paper. We model the situation where the boundary acts as an attractor for the process as evidenced by a large proportion of the historical process values at or near the lower boundary. It could be interesting to develop a continuous time model that can accommodate this feature. Another possibility is to consider continuous time processes with reflections such as Skorokhod equations. The model introduced in this paper need not only be considered for price processes but can be applied to indices that are presumed to be bounded such as volatility indices. The dimensionality of the model may also be adjusted based on the dependency of the underlying historical process. We leave these matters for future research.

There are many other unanswered questions that we leave for future research as well. In particular, we haven't considered the economic driving forces of exchange rate mechanisms and their impact on the rate dynamics.

\section{Acknowledgements}

This work was supported by the ARC grant of Australia DP120100928. We would like to thank an anonymous reviewer for suggestions that have been included in this paper.

\section{References}

[1] Frankel, J. A. (1999). 'No Single Currency Regime is Right for All Countries or At All Times'. Working Paper 7338 National Bureau of Economic Research. 
[2] Dokuchaev, N. (2006). 'Speculative Opportunities for Currency Exchange Under Soft Peg'. Applied Financial Economics Letters 2(6):371-374. doi:10.1080/17446540600706809.

[3] Dokuchaev, N. (2007). 'Mean-Reverting Market Model: Speculative Opportunities and NonArbitrage'. Applied Mathematical Finance 14(4):319-337. doi:10.1080/13504860701255078.

[4] Dokuchaev, N. (2012). 'Mean-Reverting Discrete Time Market Models: Speculative Opportunities and Absence of Arbitrage'. IMA Journal of Management Mathematics 23(1):17-27. doi: 10.1093/imaman/dpq015.

[5] Carcano, G.; Falbo, P. and Stefani, S. (2005). 'Speculative Trading in Mean Reverting Markets'. European Journal of Operational Research 163(1):132-144. ISSN 0377-2217. doi: 10.1016/j.ejor.2004.01.002.

[6] BAchelier, L. (1900). 'Théorie de la Spéculation'. Annales de l'Ecole Normale Supérieure 17:21-86.

[7] Kendall, M. G. (1953). 'The Analysis of Economic Time-Series. Part 1. Prices'. Journal of the Royal Statistical Society 96:11-25.

[8] Shiryaev, A. N. (1999). Essentials of Stochastic Finance: Facts, Models, Theory volume 3 of Advanced Series on Stastical Science \& Applied Probability. World Scientific Publishing Co. Pte. Ltd.

[9] Black, F. and Scholes, M. (1973). 'The Pricing of Options and Corporate Liability'. Journal of Political Economy 81:637-654.

[10] Mandelbrot, B. B. (1963). 'The Variation of Certain Speculative Prices'. Journal of Business 36:394-419.

[11] FAmA, E. F. (1963). 'Mandelbrot and the Stable Paretian Hypothesis'. Journal of Business 36:420429.

[12] McFarland, J. W.; Pettit, R. R. and Sung, S. K. (1982). 'The Distribution of Foreign Exchange Price Changes: Trading Day Effects and Risk Measurement'. The Journal of Finance 37(3):693-715.

[13] Blattberg, R. C. and Gonedes, N. (1974). 'A Comparison of the Stable and Student Distributions as Statistical Models for Stock Prices'. Journal of Business 47:244-280.

[14] Boothe, P. and Glassman, D. (1987). 'The Statistical Distribution of Exchange Rates'. Journal of International Economics 22:297-319.

[15] Hsien, D. A. (1988). 'The Statistical Properties of Daily Foreign Exchange Rates: 1974-1983'. Journal of International Economics 24:129-145.

[16] Baillie, R. T. and Bollerslev, T. (2002). 'The Message in Daily Exchange Rates: A ConditionalVariance Tale'. Journal of Business $\& 3$ Economic Statistics 20(1):60-68.

[17] Müller, U. A.; Dacorogna, M. M.; Olsen, R. B.; Pictet, O. V.; Schwarz, M. and MorGENEGG, C. (1990). 'Statistical Study of Foreign Exchange Rates, Empirical Evidence of a Price Change Scaling Law, and Intraday Analysis?' Journal of Banking and Finance 14:1189-1208.

[18] Los, C. A. and Karuppiah, J. (2002). 'Wavelet Multiresolution Analysis of High-Frequency Asian FX Rates, Summer 1997'. Adelaide University, School of Economics Working Paper 00-6 AFA 2001 New Orleans. doi:10.2139/ssrn.245744.

[19] Krugman, P. R. (1991). 'Target Zones and Exchange Rate Dynamics'. Quarterly Journal of Economics 106:669-82.

[20] Hui, C. H. and Fong, T. (2007). 'Is the Hong Kong Dollar Exchange Rate 'Bounded' in the Convertibility Zone?' Working Paper 13 Hong Kong Monetary Authority. doi:10.2139/ssrn.1019432.

[21] Yee, H. B. (2015). 'On the Impact of the Boundary on Dynamics: anti-persistence in the case of the HKD exchange rate corridor'. Annals of Financial Economics 10(1):forthcoming. 
[22] Nicolau, J. (2002). 'Stationary Processes that look like Random Walks - The Bounded Random Walk Process in Discrete and Continuous Time'. Econometric Theory 18(1):99-118.

[23] Dokuchaev, N. (2014). 'Volatility Estimation from Short Time Series of Stock Prices'. Journal of Nonparametric Statistics 26(2):373-384. doi:10.1080/10485252.2013.844805.

[24] Zhou, B. (1996). 'High-Frequency Data and Volatility in Foreign-Exchange Rates'. Journal of Business \&3 Economic Statistics 14(1):45-52.

[25] Feller, W. (1971). An Introduction to Probability Theory and its Applications. Wiley Series in Probability and Mathematical Statistics: Probability and Mathematical Statistics. Wiley. ISBN 9780471257097. 